\title{
Influence of habitat features of urban streetscapes on richness and abundance of avian species
}

\author{
Sarbasis DutTA ${ }^{1,3}$, Goutam Kumar SAHA ${ }^{1} \&$ Subhendu \\ MAZUMDAR ${ }^{2, *}$
}

Received: March 08, 2021 - Revised: May 19, 2021 -Accepted: May 20, 2021

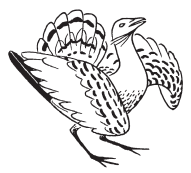

Dutta, S., Saha, G. K. \& Mazumdar, S. 2021. Influence of habitat features of urban streetscapes on richness and abundance of avian species. - Ornis Hungarica 29(1): 20-32. DOI: 10.2478/ orhu-2021-0002

\begin{abstract}
In human-dominated landscapes, roads are known to negatively influence birds causing decline in species richness, as well as reduction in the number of avian species. However, linear stretches of green spaces formed by roadside plantations in urban streetscapes can support diverse avian communities. In spite of being an integral habitat feature of urban areas, there is a clear paucity of studies on avian diversity in urban streetscapes. The present study was carried out in Kolkata, where data on avian species richness and abundance was collected from 16 randomly placed belt transects (replicates), each of $500 \mathrm{~m}$ length and $20 \mathrm{~m}$ width, on different major roads throughout the study area keeping a minimum gap of $200 \mathrm{~m}$ between adjacent transects to avoid data overlapping. Each of these transects were traversed on foot twice in a month from January to March 2017 during days with calm weather conditions. We recorded 31 species of birds belonging to 8 orders and 19 families, of which maximum species belonged to the order Passeriformes (13 species). We found that both abundance and species richness of birds in transects with higher number of trees ( $78 \pm 4.1$ individuals and $19.55 \pm 1.703$ species of birds) were significantly higher than transects with fewer trees (53.74 2.5 individuals and $9.5 \pm 0.789$ species of birds). Amongst various habitat features along these streetscapes, the total number of trees positively influenced both species richness (GLMM: $\mathrm{F}_{1,90}=14.485, \mathrm{P}<0.05$ ) and abundance of birds (GLMM: $\mathrm{F}_{1,90}=8.081, \mathrm{P}<0.05$ ). However, the other land use variables (i.e. number of bushes, waterbodies, markets and buildings) neither influenced the abundance of birds nor the species richness. Our findings can be useful for urban development to perceive the importance of various habitat features in urban streetscapes in sustaining avian diversity.
\end{abstract}

Keywords: avifauna, species richness, streetscape, roadside vegetation, habitat, urbanization

Összefoglalás Az emberi tevékenység révén átalakított környezetben az utak negatív hatással vannak a madarakra, csökkentik a fajok számát és a populációk egyedszámát. Ezzel együtt az utak mentén kialakított zöld növényzeti sávok diverz madárközösségnek adhatnak otthont. Habár az útmenti növényzet a városi élőhely integráns része, ritkán vizsgálják az itt előforduló madarakat. E vizsgálatban Kalkutta fơútjai mentén 16, egyenként $500 \mathrm{~m}$ hosszú és $20 \mathrm{~m}$ széles transzektben vizsgáltuk az előforduló madárfajok számát és denzitását. A madárszámolásokat gyalog végeztük 2017. január és március között, transzektenként 6 alkalommal. Összesen 31 fajt ( 8 rendböl, 19 családból) figyeltünk meg, melyek nagy része énekesmadár volt (Passeriformes). Azokban a transzektekben, amik mentén sok fa található, a madarak denzitása és fajszáma is nagyobb volt $(78 \pm 4,1$ egyed $19,55 \pm 1,703$

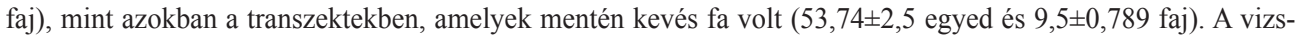
gált élöhelyi tulajdonságok közül kizárólag a fák száma volt hatással a madarak denzitására (GLMM: $\mathrm{F}_{1,90}=8,081$, $\mathrm{P}<0,05$ ) és a fajszámra (GLMM: $\mathrm{F}_{1,90}=14,485, \mathrm{P}<0,05$ ). A többi változó, mint a bokrok száma, víztestek, piacok és épületek száma sem a fajszámra, sem a madarak denzitására nem volt hatással. Eredményeink hasznosak lehetnek a városfejlesztési tervek kidolgozásában, a városi élöhelyek kialakításában és így a madarak diverzitásának megörzésében.

Kulcsszavak: madárfauna, fajgazdagság, utcakép, útmenti növényzet, élőhely, urbanizáció 
${ }^{1}$ Department of Zoology, University of Calcutta, 35, Ballygunge Circular Road, Kolkata-700019, West Bengal,
India
${ }^{2}$ Department of Zoology, Shibpur Dinobundhoo Institution (College), 412/1, G.T.Road (South), Shibpur, Howrah
- 711102, West Bengal, India
${ }^{3}$ Present address: Bombay Natural History Society, Mumbai, India
${ }^{*}$ corresponding author, e-mail: subhendumazumdar@gmail.com

\section{Introduction}

Cities and towns across the globe are ever-expanding with explosion in human population (Fuller et al. 2009). As compared to the year 2000, a three-fold increase in urban areas have been predicted by 2030 (Seto et al. 2012) leading to large scale loss, degradation and fragmentation of habitats coupled with environmental changes (McKinney 2006, BarMassada et al. 2014), destruction of forests (Fischer et al. 2007), reduction of wilderness areas (Olagunju 2015) and increase in impervious surfaces (Barnes et al. 2001), which often threatens the survival of many species worldwide in these areas (Marzluff et al. 2001, Sol et al. 2017). Animals in urban areas also face greater amount of anthropogenic pressures (such as increased vehicular traffic, air, noise and light pollution, loss of vegetation cover and increased impervious surfaces). In such scenario, various native greenspaces serve as important and remnant patches of habitats for wildlife thriving in human-dominated landscapes (Miller 1997, Milton 2002). Green spaces in urban areas are often rich in biodiversity (Shwartz et al. 2014a, 2014b) and have long been identified to increase the functional connectivity for the local fauna (Ikin et al. 2015). Studies on the richness and diversity of birds have been carried out in urban greenspaces, like parks, forest remnants, cemeteries (Lussenhop 1977, Kocian et al. 2003, Croci et al. 2008, Nielsen et al. 2014).

Roads are often known to negatively influence birds by increasing impervious surfaces, leading to fragmentation and destruction of habitats; as well as increase traffic and consequent mortality being hit by speeding vehicles; elevated levels of air, noise and light pollutions etc., which often decline the abundance of various avian species (Lim \& Sodhi 2004, Cooke et al. 2020). Increasing area of roads have been reported to lead to a decline in species richness (Villasenor et al. 2017). Nevertheless, the linear stretches of green spaces formed by roadside plantations are an integral habitat feature of urban areas (Gonzalez Sosa et al. 2017), which increase the functional connectivity to the local fauna (Ikin et al. 2015). Urban streetscapes are also known to support diverse avian communities (White et al. 2005). However, there is a clear paucity of studies on avian diversity in urban streetscapes from highly populated countries with intense population explosion and rapid urbanization, like India. Therefore, we carried out this study in an urban area to (i) make an assessment of the community composition, species richness and abundance of avifauna in different streetscapes and (ii) to assess which habitat features influenced the abundance and species richness of birds. Our findings can be useful in the management of urban streetscapes sustaining the avian diversity thriving there. 


\section{Methods}

\section{Study design}

We carried out this study in Kolkata $\left(22.330^{\circ} \mathrm{N}, 88.300^{\circ} \mathrm{E} ; 6.4 \mathrm{~m}\right.$ a.s.1.) (Figure 1), which spreads linearly along the banks of the Hooghly River and inhabited by around 4.5 million residents. This study area is located in the lower Ganges basin and one of the largest urban agglomeration in India and also of the world. Data on avian species richness and abundance was collected following the belt-transect method (Bibby et al. 2000, Sutherland 2006). 16 belt transects (replicates), each of $500 \mathrm{~m}$ length and $20 \mathrm{~m}$ width were randomly placed on different major roads throughout the study area keeping a minimum gap of $200 \mathrm{~m}$ between adjacent transects to avoid data overlapping. All transects were well dispersed from each other (Hurlbert 1984) and representative of the entire study area (Figure 1). Each of these transect was traversed on foot twice in a month from January to March 2017, which resulted in a total of six surveys per transect. Surveys were carried out during days with calm weather

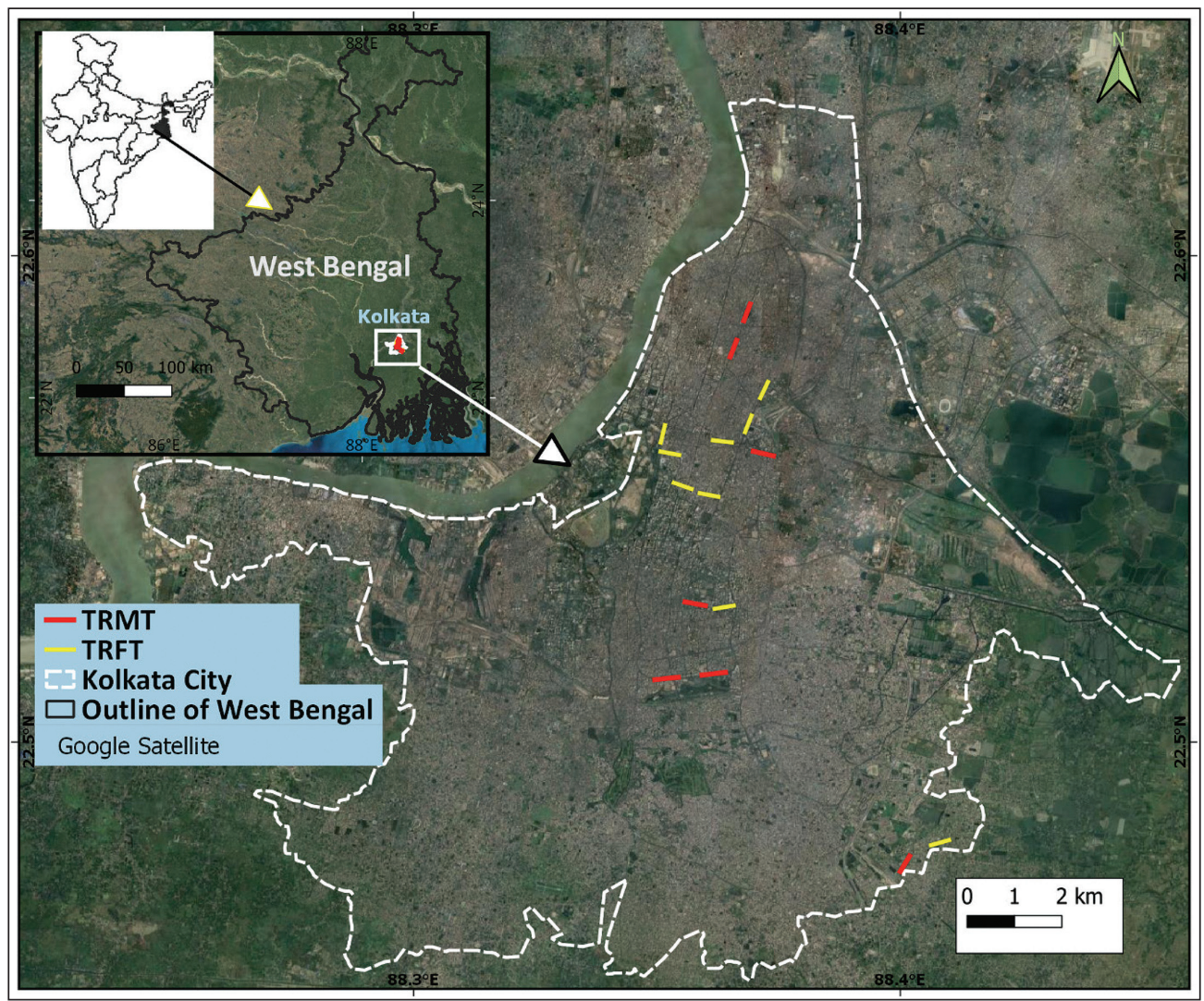

Figure 1. Map of study area in West Bengal, India showing the locations of transects with many trees (TRMT) and with few trees (TRFT)

1. ábra A vizsgálati terület térképe (Kalkutta, Nyugat-Bengál, India). A transzektek helyét piros (transzekt sok fával) és sárga (transzekt kevés fával) vonalak jelölik 
conditions (without rain and strong wind) and during morning hours (between 06:00 and 09:30), when birds are usually most active. While traversing the transect, we collected data on the abundance and species richness of the avifauna. Any bird noticed horizontally $10 \mathrm{~m}$ on either side of transects were recorded, as well as, the individuals within $10 \mathrm{~m}$ height were recorded. Flying birds were recorded only to get a 'snap-shot' of all the birds recordable from the transect (Bibby et al. 2000). However, the 'fly through' and 'fly over' individuals were excluded from subsequent analyses as they would produce overestimates (Bibby et al. 2000). Birds were observed either with unaided eyes or with the help of a pair of binoculars (Nikon $8 \times 40$ ) and photographs were taken with a digital camera (Nikon D500 Digital SLR Camera) for documentation. Birds were identified and their migratory status (resident/ migratory) were determined using field guides (Grimmett et al. 2016). We also calculated the percentage occurrence score of each species to assess their local abundance, where very common (Vc) bird species were recorded on $80-100 \%$ of field visits, common (Co) species on $50-79 \%$ of field visits, fairly common (Fc) on $20-49 \%$ of field visits and rare (Ra) on less than $20 \%$ of the field visits (Khan \& Naher 2009). The conservation status of birds and their global population trend were taken from the IUCN Red List (del Hoyo et al. 2014). Feeding guild is defined as a group of species with similar foraging habits (Hutto 1985). Our observed avian species were divided into seven guilds, i.e. carnivore (Car), omnivore (Omn), frugivore (Frug), herbivore (Herb), nectarivore (Nect), granivore (Gran) and insectivore (Ins), following Ali and Ripley (1987).

In urban areas, trees (White et al. 2005), canopy cover (Alberti \& Marzluff 2004, MacGregor-Fors \& Schondube 2011), bushes/greenspaces (Ortega-Álvarez \& MacGregorFors 2009), presence of waterbodies (Johnson et al. 2012) and building density (Germaine et al. 1998) and other urban structures (Ortega-Álvarez \& MacGregor-Fors 2009) are known to potentially influence the diversity and abundance of avian communities present there. Hence, the habitat features like numbers of trees, bushes, waterbodies (any permanent water sources like inland waterbodies, pond, artificial, natural lake, canal which potentially influence bird abundance), buildings and markets (permanent commercial places demarcated by municipal corporation) present within each of the belt transects were assessed from the cloud free high-resolution satellite image of Kolkata (Image acquisition: 24.11.16) obtained from Google Earth Pro software (ver. 7.3.3.7699), which was confirmed through rigorous ground-truthing on field. These were used as habitat features of each transect.

\section{Data analysis}

Non-parametric tests were performed for data analysis as Shapiro-Wilk's tests revealed that the abundance $(\mathrm{W}=0.972, \mathrm{df}=96, \mathrm{P}=0.036)$ and species richness $(\mathrm{W}=0.782, \mathrm{df}=96, \mathrm{P}<0.05)$ were non-normally distributed. The mean value of trees in all 16 transects were $9.69 \pm 1.34$. Therefore, we used presence of 10 trees per $500 \mathrm{~m}$ as threshold for classifying the transects into two broad categories like (i) transects with few trees (i.e. $<10$ trees $/ 500 \mathrm{~m}$; henceforth TRFT) and (ii) transects with many trees (i.e. $>10$ trees $/ 500 \mathrm{~m}$; henceforth TRMT). Then Mann-Whitney U test was applied to find out if the species richness and abundance of birds showed any significant variation between TRFT and TRMT. 


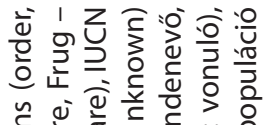

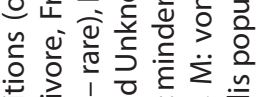

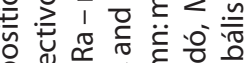
¿ 는 है ช

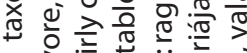

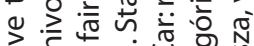
过

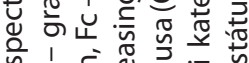

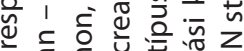

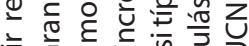

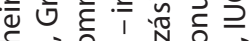

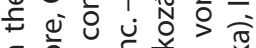

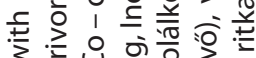

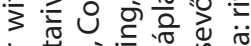

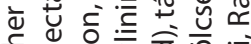

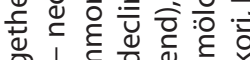
ช 1 है ब है

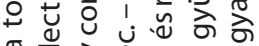

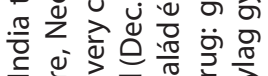

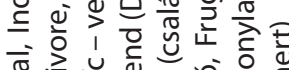

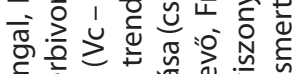
बे

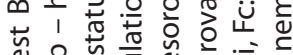

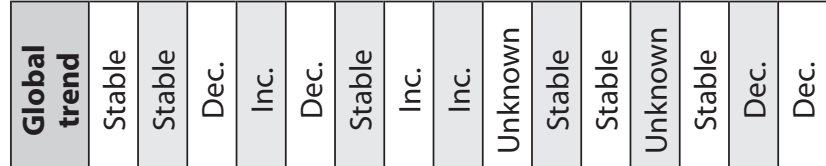

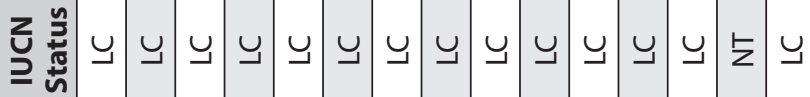

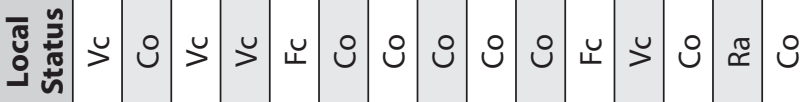
㐫

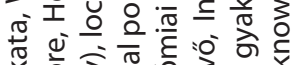

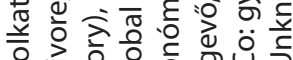
a

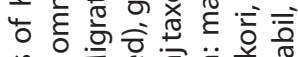
管

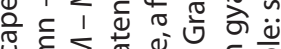

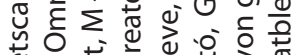

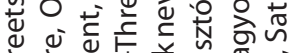

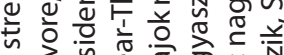

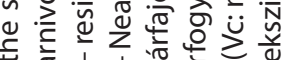
\#

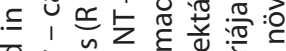

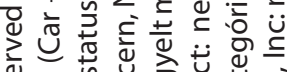

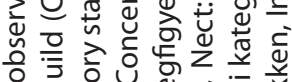
年

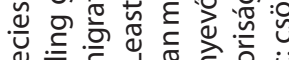

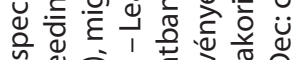

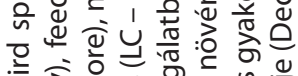
言

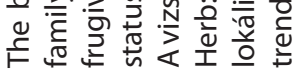

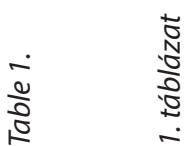

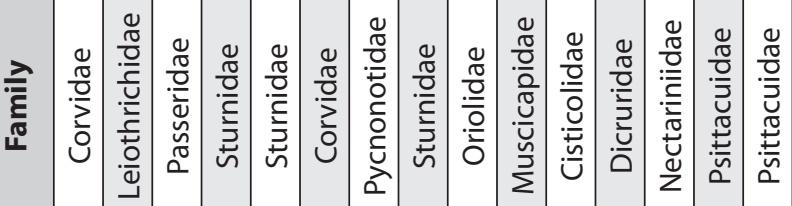

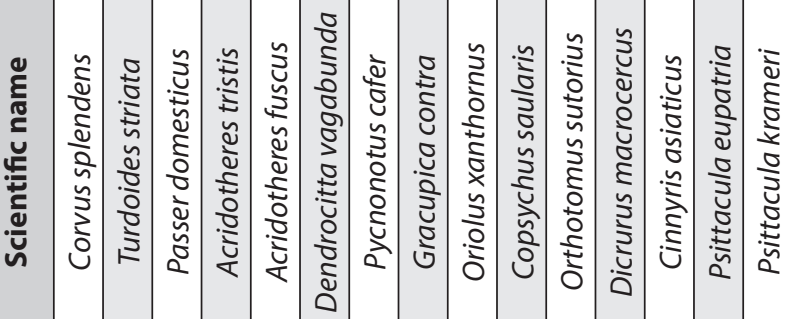

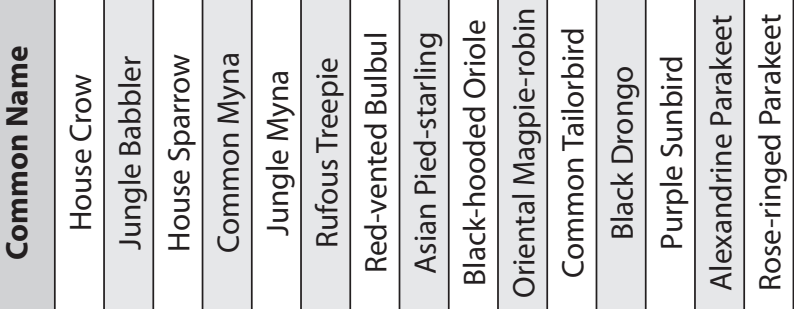




\begin{tabular}{|c|c|c|c|c|c|c|c|c|c|c|c|c|c|c|c|c|}
\hline 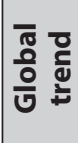 & $\stackrel{\dot{\varphi}}{\underline{S}}$ & 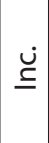 & $\mid \begin{array}{l}\frac{0}{0} \\
\frac{0}{0} \\
\sim \\
\sim\end{array}$ & $\begin{array}{l}\frac{0}{0} \\
\frac{\pi}{\pi} \\
\sim\end{array}$ & هั & $\begin{array}{l}\frac{0}{0} \\
\frac{\pi}{\pi}\end{array}$ & $\underline{\text { ک }}$ & ن. & 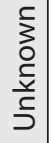 & $\stackrel{\dot{\dot{ }}}{\subseteq}$ & $\left|\begin{array}{l}\frac{5}{3} \\
0 \\
\frac{c}{5} \\
\frac{5}{J}\end{array}\right|$ & $\begin{array}{l}\frac{5}{3} \\
0 \\
\frac{c}{c} \\
\frac{\mathbf{c}}{J} \\
\end{array}$ & $\stackrel{\dot{v}}{\subseteq}$ & ù & هั & Uू. \\
\hline 늘 & ב & $\cup$ & $\cup$ & $\cup$ & $\cup$ & U & $\cup$ & 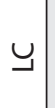 & $\cup$ & $\cup$ & $\cup$ & $\cup$ & $\cup$ & $\cup$ & $\cup$ & $\supsetneq$ \\
\hline 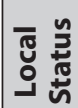 & ن & $\mathscr{\widetilde { I }}$ & प्u & ن & U్ & త & प्山 & 氐 & uㅗ & ن & $\stackrel{\pi}{\simeq}$ & $\mathscr{\pi}$ & 0 & $\stackrel{u}{>}$ & O & $\ddot{\mathscr{x}}$ \\
\hline 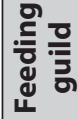 & 인 & 온 & 인 & $\underline{\varrho}$ & $\cong$ & 气 & $\bar{U}$ & ن্ত & 㐫 & 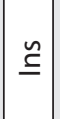 & 㐫 & U & 䏓 & $\left|\begin{array}{l}\frac{5}{0} \\
\frac{0}{0}\end{array}\right|$ & $\cong$ & 㐫 \\
\hline $\begin{array}{l}\frac{0}{0} \\
\text { ò }\end{array}$ & & & & 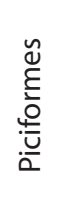 & & & & $\begin{array}{l}\tilde{\varrho} \\
\frac{0}{0} \\
\stackrel{0}{0} \\
\frac{\pi}{0}\end{array}$ & & & 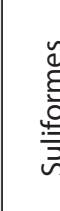 & & ह & 总 & 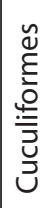 & 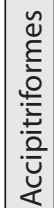 \\
\hline 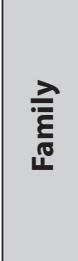 & 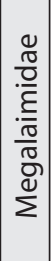 & 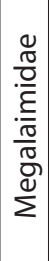 & 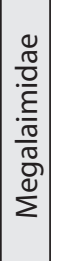 & $\begin{array}{l}\frac{\pi}{0} \\
\frac{\pi}{0} \\
\frac{0}{2}\end{array}$ & $\begin{array}{l}\frac{\pi}{0} \\
\frac{\pi}{0} \\
\frac{0}{2} \\
\end{array}$ & $\begin{array}{l}\frac{\pi}{\pi} \\
\frac{\pi}{U} \\
\frac{0}{2}\end{array}$ & 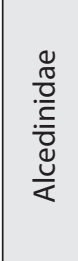 & 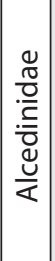 & 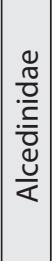 & 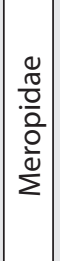 & 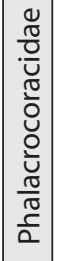 & 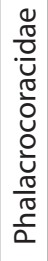 & $\left|\begin{array}{c}0 \\
\frac{0}{0} \\
\frac{0}{0} \\
\frac{\varepsilon}{5} \\
\frac{3}{0} \\
0\end{array}\right|$ & 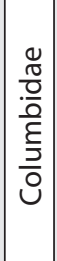 & 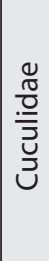 & 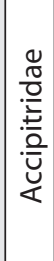 \\
\hline 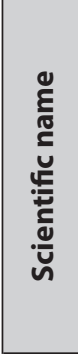 & 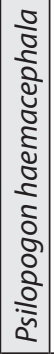 & 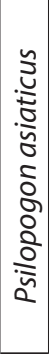 & 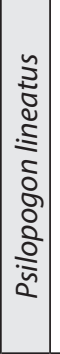 & 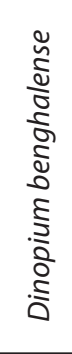 & 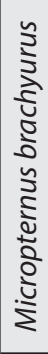 & 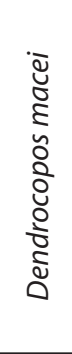 & 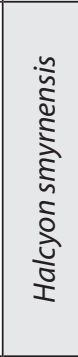 & 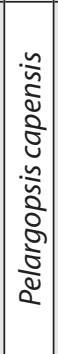 & 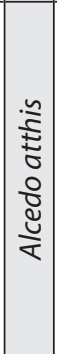 & 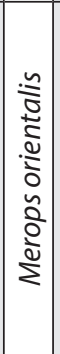 & 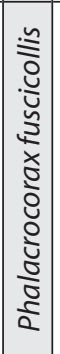 & 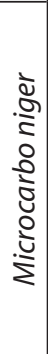 & 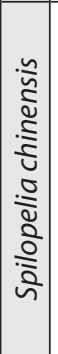 & 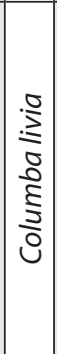 & 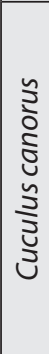 & 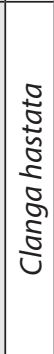 \\
\hline 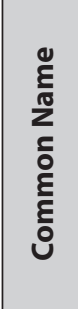 & 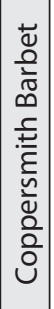 & 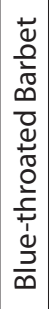 & 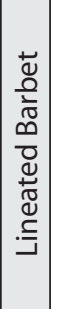 & 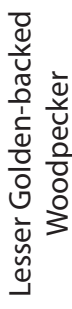 & 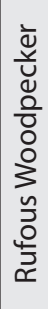 & 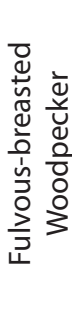 & 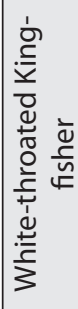 & 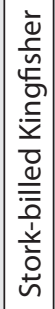 & 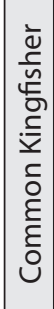 & 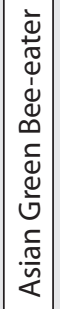 & 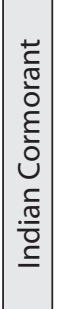 & 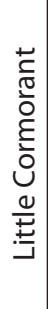 & 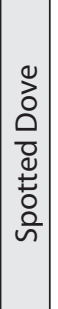 & 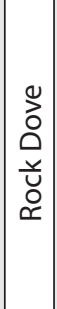 & 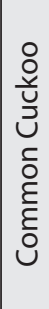 & 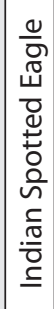 \\
\hline
\end{tabular}


We found that the distribution of species richness and abundance were over-dispersed and negatively skewed (variance $>$ mean). Hence, two Generalized Linear Mixed Models (GLMMs) with negative binomial distribution and log-link were carried out separately considering species richness and abundance of birds as response variables against the habitat features (i.e. total number of trees, bushes, waterbodies, buildings and market) as predictor variables (fixed factors) and transect ID (as a random factor) in both the models. Prior to running the GLMMs, we tested multicollinearity between variables using variance inflation factor (VIF) method (Zuur et al. 2013) and only included the predictor variables with a VIF value $<5$ (Montgomery \& Peck 1992) to ensure that no variables were strongly correlated. Statistical tests were performed using SPSS software (ver. 20). Significance was tested at $p$ $<0.05$ and data were presented as mean \pm standard error.

\section{Results}

A total of 31 species of birds belonging to eight orders and 19 families were recorded during the study (Table 1), of which most species belonged to the order Passeriformes (13 species) followed by the order Piciformes (6 species). Most of the recorded species were resident (96.55\%). Among all avian species observed during the study, only the Indian Spotted Eagle Clanga hastata fall under the IUCN vulnerable (VU) category, while the remaining species are categorized as least concern (LC) species (del Hoyo et al. 2014). Assessment of local abundance revealed that out of 31 species recorded, five species $(16.1 \%)$ were very common, 14 species $(45.2 \%)$ were common, six species $(19.4 \%)$ were fairly common and six species (19.4\%) were rare. When this local abundance was compared with the global population trend for the species (del Hoyo et al. 2014), we found that two species having a globally declining trend were still very common in the study area (Table 1).

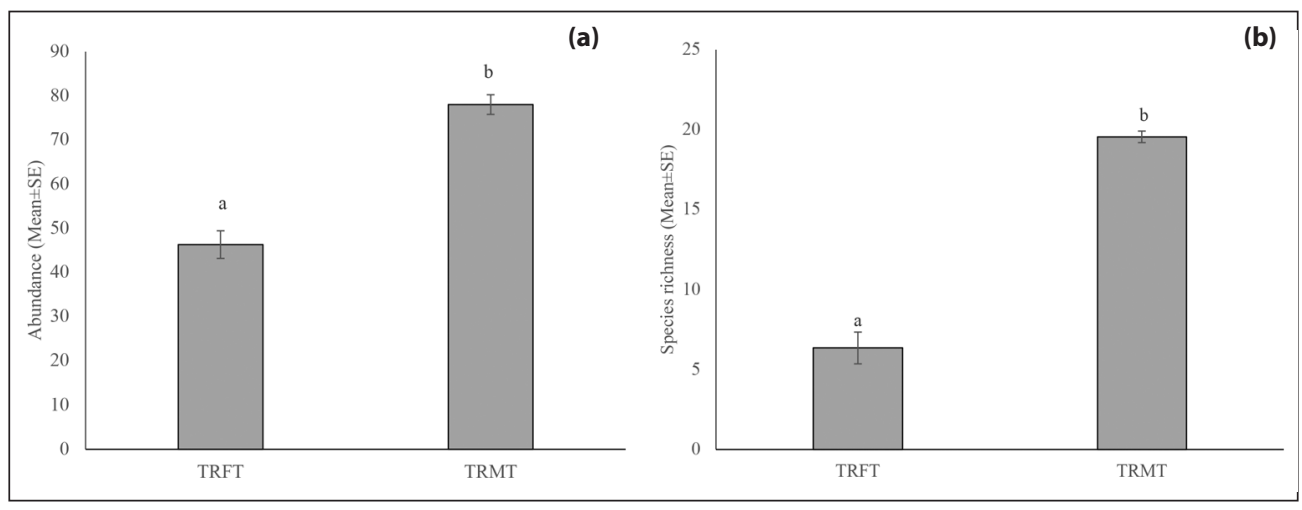

Figure 2. (a) Abundance and (b) species richness of birds in TRFT and TRMT [Columns with different letters indicate significant difference (Bonferroni post hoc tests $\mathrm{P}<0.05$ ). Error bars indicate standard errors (SE) of means]

2. ábra A madarak (a) denzitása és (b) fajszáma a kevés (TRFT) és sok (TRMT) sok fát tartalmazó transzektek mentén. Az oszlopok magassága és a vonalak az átlagot \pm SE mutatják, az oszlopok fölötti eltérő betűk a szignifikáns különböző átlagot jelzik ( $P<0,05$, Bonferroni post hoc teszt) 
Table 2. Variables in the GLMMs describing the species richness and abundance of birds in streetscapes of Kolkata, West Bengal, India

2. táblázat Azélőhelyi változók kapcsolata a transzektekmentén mért fajgazdagsággal és denzitással. A táblázat az elemzéshez használt lineáris kevert modell (GMML) eredményét mutatja

\begin{tabular}{|c|c|c|c|c|c|c|c|c|}
\hline \multirow{2}{*}{ Variables } & \multicolumn{4}{|c|}{ Species richness } & \multicolumn{4}{c|}{ Abundance } \\
\cline { 2 - 10 } & $\mathbf{F}$ & $\mathbf{d f 1}$ & $\mathbf{d f 2}$ & $\mathbf{P}$ & $\mathbf{F}$ & $\mathbf{d f 1}$ & $\mathbf{d f 2}$ & $\mathbf{P}$ \\
\hline Number of trees & $\mathbf{1 4 . 4 8 5}$ & $\mathbf{1}$ & $\mathbf{9 0}$ & $\mathbf{0 . 0 0 0}$ & $\mathbf{8 . 0 8 1}$ & $\mathbf{1}$ & $\mathbf{9 0}$ & $\mathbf{0 . 0 0 6}$ \\
\hline Number of bushes & 1.238 & 1 & 90 & 0.269 & 2.81 & 1 & 90 & 0.097 \\
\hline Number of waterbodies & 1.415 & 1 & 90 & 0.237 & 1.796 & 1 & 90 & 0.184 \\
\hline Number of markets & 0.152 & 1 & 90 & 0.697 & 1.45 & 1 & 90 & 0.232 \\
\hline Number of buildings & 1.312 & 1 & 90 & 0.255 & 0.051 & 1 & 90 & 0.822 \\
\hline
\end{tabular}

Out of all species of birds recorded during this study, only two species (Stork-billed Kingfisher Pelargopsis capensis and Lineated Barbet Psilopogon lineatus) were found exclusively in the TRMT and 29 species (93.55\%) were found in both TRFT and TRMT. We also found that the abundance of birds in TRMT (78 \pm 4.1 individuals) were significantly higher (Mann Whitney test: $\mathrm{U}=189, \mathrm{P}=0.000$ ) than the abundance of birds recorded from TRFT (53.74 \pm 2.5 individuals) as shown in Figure $2 a$. Similarly, Figure $2 b$ shows that the species richness of TRMT (19.55 1.7 species) was also significantly higher (Mann Whitney test: $\mathrm{U}=200.5, \mathrm{P}=0.000)$ than TRFT $(9.5 \pm 0.8$ species $)$. GLMM further revealed that the species richness $\left(\mathrm{F}_{1,90}=14.485, \mathrm{P}<0.05\right)$ and the abundance of birds $\left(\mathrm{F}_{1,90}=8.081, \mathrm{P}<0.05\right)$ were positively influenced by the number of trees (Table 2). However, the other land use variables (i.e. number of bushes, waterbodies, markets and buildings) neither influenced the abundance of birds nor the species richness (Table 2).

\section{Discussion}

Urban areas across the globe are inhabited by many species of flora and fauna (Shwartz et al. 2014a, 2014b), including birds (Gatesire et al. 2014). Birds are often abundant in areas with suitable survival conditions (Veech et al. 2010) and the structural features of any habitat give important cues for birds to decide upon whether to use that particular habitat or not (Cody 1981). Greenspaces with suitable structure and floral composition favour bird communities in urban areas (Jokimäki 1999, Daniels \& Kirkpatrick 2006, MacGregor-Forz et al. 2009) and areas with rich vegetation in the greenspaces usually sustain greater bird abundance as compared to areas with impoverished vegetation (Chace \& Walsh 2006). We also found that species richness and the abundance of birds were higher in TRMT than in TRFT. Moreover, amongst various land use features, the increasing number of trees also increased the species richness and abundance of birds during the present study. Several authors found that trees in urban areas usually attract the moderately abundant species (Jokimäki 1999, Ortega-Álvarez \& MacGregor-Fors 2009, MacGregor-Fors et al. 2010). Few others mentioned that sufficient number of greenspaces in urban areas may even support forest birds (Mortberg \& Wallentias 2000, Park \& Lee 2000). Again, Sandstrom et 
al. (2006) emphasized the importance of large trees and multi-layered vegetation for urban avian richness. Increasing tree cover provides crucial resources for the arboreal and forest birds (Ciach \& Frohlich 2017).

Urban bird communities highly depend on the structure and type of vegetation (White et al. 2005). Abundance of bird species are positively influenced by the richness of native tree species (Chace \& Walsh 2006, Paker et al. 2014). Particularly the native species that existed prior to urbanization are largely benefitted by the plantation of various native tree species (Bhullar \& Majer 2000). As compared to exotic trees, the diversity and abundance of insects as well as other resources for birds (such as fruits, nectar etc) are often higher in native trees (White et al. 2005, Ikin et al. 2013). The indigenous species also support greater number of arthropods (Bhullar \& Majer 2000) and therefore, are frequented by large number of insectivorous birds (Majer et al. 1994, Recher et al. 1996). During the present study, we also noticed several old and native trees (such as Banyan, Peepal etc.) which supported great number of birds. However, due to paucity of scientific studies, exotic tree species are arbitrarily planted along the roadside in many urban areas. Thus, implementation of effective strategies and incentives that encourage the planting of native vegetation in streetscapes and garden should be paramount (White et al. 2005). Protection of large indigenous trees along the roads, plantation of a greater number of native trees and retaining the existing bushes will undoubtedly be beneficial to sustain the bird communities in the urban landscape (White et al. 2005).

Water birds (like ducks, waders and piscivores) and other wetland associated birds (like kingfishers) are mainly benefitted by increasing water area (Yuan et al. 2014) as they often aggregate around such water bodies. Chamberlain et al. (2007) reported that the presence of waterbodies in urban areas increased avian species richness. However, only three species of kingfishers (White-throated Kingfisher Halcyon smyrnensis, Stork-billed Kingfisher Pelargopsis capensis and Common Kingfisher Alcedo atthis) and two species of cormorants (Indian Cormorant Phalacrocorax fuscicollis and Little Cormorant Microcarbo niger) were rarely noticed during the present study. Possibly, due to such rare presence of water birds in our study area, we did not find any influence of water bodies on the abundance and species richness of birds.

Bushes are important mainly for forest-dwelling birds and urban avoiders to thrive in the human dominated habitats (Brandt et al. 2013, Gopal et al. 2018) and increasing number of buildings have been reported to decrease species richness (Evans et al. 2009). Few species of urban exploiters and scavengers congregate around the markets and its adjacent garbage dumping sites (Mazumdar et al. 2016, 2018, 2019) and forage on the refuse as these foods are plenty and predictable. However, we did not notice any significant influence of bushes, markets and buildings on the species richness and abundance of birds.

Our findings indicate that plantation of indigenous trees along the roads might be beneficial in sustaining greater avian diversity in urban streetscapes. Particularly in urban areas of developing countries, which are rapidly losing the greenspaces due to infrastructure development, the roadside plantations might serve as important habitat for birds. Street trees in urban areas are also associated with higher property values, reduced crime rates, economic benefits (Abd Kadir \& Othman 2012), as well as are known to perform various 
important ecological roles (Bhullar \& Majer 2000). They provide manifold benefits such as absorption of GHGs, reduction of air pollution due to vehicular exhausts (Johnson 2009), watershed protection, providing shade on asphalt and concrete structures thereby, reducing the ambient air temperature (Abd Kadir \& Othman 2012). However, sometimes falling of street trees lead to casualties and damage of properties, particularly after storms. Falling of large trees in urban areas often happen due to unplanned developmental activities around the root area of the trees (such as random and unplanned cutting the roots for various infrastructural modifications, higher abundance of rodents or termites around the tree roots making the root loosening the soil around the root system etc.). Plantation of appropriate roadside trees will be useful to sustain and also elevate the avian diversity in urban areas, as well as increase the aesthetic value of citizens. Empirical evidences of this research can be useful for urban planners to perceive the importance of various habitat features in urban streetscapes in sustaining the avian diversity. The managers and wildlife planners need to realize the importance of the streetscapes in conservation of urban avian diversity.

\section{Acknowledgement}

We thank Mr. Souvik Barik for preparing the study area map. We are indebted to the Principal, Shibpur Dinobundhoo Institution (College) and Head of the Department of Zoology of the University of Calcutta for providing necessary infrastructural facilities. We also acknowledge the constructive inputs from Dr. Tibor Csörgö and two unknown reviewers in improving this manuscript.

\section{References}

Abd Kadir, M. A. \& Othman, N. 2012. Towards a better tomorrow: street trees and their values in urban areas. Procedia-Social and Behavioral Sciences 35: 267-274. DOI: 10.1016/j.sbspro.2012.02.088

Alberti, M. \& Marzluff, J. M. 2004. Ecological resilience in urban ecosystems: linking urban patterns to human and ecological functions. - Urban Ecosystems 7(3): 241-265. DOI: 10.1023/B:UECO.0000044038.90173.c6

Ali, S. \& Ripley, S. D. 1987. Compact Handbook of birds of India and Pakistan together with those of Bangladesh, Nepal, Bhutan, and Srilanka. - Oxford University Press, Delhi

Bar-Massada, A., Radeloff, V. C. \& Stewart, S. I. 2014. Biotic and abiotic effects of human settlements in the wildland-urban interface. - BioScience 64(5): 429-437. DOI: 10.1093/biosci/biu039

Barnes, K. B., Morgan, J. \& Roberge, M. 2001. Impervious Surfaces and the Quality of Natural and Built Environments. - Baltimore: Department of Geography and Environmental Planning, Towson University. Retrieved from: https://citeseerx.ist.psu.edu/viewdoc/download?doi=10.1.1.477.2834\&rep=rep1\&type=pdf

Bhullar, S. \& Majer, J. 2000. Arthropods on Street Trees: A Food Resource for Wildlife. - Pacific Conservation Biology 6: 171-173. DOI: 10.1071/PC000171

Bibby, C. J., Burgess, N. D., Hill, D. A. \& Mustoe, S. 2000. Bird Census Techniques. $2^{\text {nd }}$ ed. - Elsevier, DOI: 10.1016/C2009-0-03531-4

Brandt, J. S., Wood, E. M., Pidgeon, A. M., Han, L-X., Fang, Z. \& Radeloff, V. C. 2013. Sacred forests are keystone structures for forest bird conservation in southwest China's Himalayan Mountains. - Biological Conservation 166: 34-42. DOI: 10.1016/j.biocon.2013.06.014

Chace, J. F. \& Walsh, J. J. 2006. Urban effects on native avifauna: a review. - Landscape and Urban Planning74(1): 46-69. DOI: 10.1016/j.landurbplan.2004.08.007

Chamberlain, D. E., Gough, S., Vaughan, H., Vickery, J. A. \& Appleton, G. F. 2007. Determinants of bird species richness in public green spaces. - Bird Study 54(1): 87-97. DOI: 10.1080/00063650709461460 
Ciach, M. \& Fröhlich, A. 2017. Habitat type, food resources, noise and light pollution explain the species composition, abundance and stability of a winter bird assemblage in an urban environment. - Urban Ecosystems 20(3): 547-559. DOI: 10.1007/s11252-016-0613-6

Cody, M. L. 1981. Habitat selection in birds: the roles of vegetation structure, competitors, and productivity. BioScience 31(2): 107-113. DOI: 10.2307/1308252

Cooke, S. C., Balmford, A., Johnston, A., Newson, S. E. \& Donald, P. F. 2020. Variation in abundances of common bird species associated with roads. - Journal of Applied Ecology 57(7): 1271. DOI: 10.1111/13652664.13614

Croci, S., Butet, A., Georges, A., Aguejdad, R. \& Clergeau, P. 2008. Small urban woodlands as biodiversity conservation hot-spot: a multi-taxon approach. - Landscape Ecology 23(10): 1171-1186. DOI: 10.1007/ s10980-008-9257-0

Daniels, G. D. \& Kirkpatrick, J. B. 2006. Does variation in garden characteristics influence the conservation of birds in suburbia? - Biological Conservation 133(3): 326-335. DOI: 10.1016/j.biocon.2006.06.011

del Hoyo, J., Collar, N. J, Christie, D. A., Elliott, A. \& Fishpool, L. D. C. 2014. Illustrated Checklist of the Birds of the World. - Lynx Edicions, Spain and BirdLife International, Cambridge

Evans, K. L., Gaston, K. J., Sharp, S. P., McGowan, A., Simeoni, M. \& Hatchwell, B. J. 2009. Effects of urbanisation on disease prevalence and age structure in Blackbird Turdus merula populations. - Oikos 118(5): 774-782. DOI: 10.1111/j.1600-0706.2008. 17226.x

Fischer, J. \& Lindenmayer, D. B. 2007. Landscape modification and habitat fragmentation: a synthesis. Global Ecology and Biogeography 16(3): 265-280. DOI: 10.1111/j.1466-8238.2007.00287

Fuller, R. A. \& Gaston, K. J. 2009. The scaling of green space coverage in European cities. - Biology Letters 5(3): 352-355. DOI: 10.1098/rsbl.2009.0010

Gatesire, T., Nsabimana, D., Nyiramana, A., Seburanga, J. L. \& Mirville, M. O. 2014. Bird diversity and distribution in relation to urban landscape types in Northern Rwanda. - The Scientific World Journal 2014: Article ID 157824. DOI: 10.1155/2014/157824

Germaine, S. S., Rosenstock, S. S., Schweinsburg, R. E. \& Richardson, W. S. 1998. Relationships among breeding birds, habitat, and residential development in Greater Tucson, Arizona. - Ecological Applications 8(3): 680-691. DOI: 10.1890/1051-0761(1998)008[0680: RABBHA]2.0.CO;2

Gonzalez-Sosa, E., Braud, I., Becerril Piña, R., Mastachi Loza, C. A., Ramos Salinas, N. M. \& Chavez, C. V. 2017. A methodology to quantify ecohydrological services of street trees. - Ecohydrology and Hydrobiology 17: 190-206. DOI: 10.1016/j.ecohyd.2017.06.004

Gopal, D., von der Lippea, M. \& Kowarik, I. 2018. Sacred sites as habitats of culturally important plant species in an Indian megacity. - Urban Forestry \& Urban Greening 32: 113-122. DOI: 10.1016/j.ufug.2018.04.003

Grimmett, R., Inskipp, C. \& Inskipp, T. 2016. Birds of the Indian Subcontinent: India, Pakistan, Sri Lanka, Nepal, Bhutan, Bangladesh and the Maldives. - Bloomsbury Publishing

Hurlbert, S. H. 1984. Pseudo replication and the design of ecological field experiments. - Ecological Monographs 54(2): 187-211. DOI: 10.2307/1942661

Hutto, R. L. 1985. Habitat Selection in Birds. - Academic Press, Inc., Montana

Ikin, K., Knight, E., Lindenmayer, D. B., Fischer, J. \& Manning, A. D. 2013. The influence of native versus exotic streetscape vegetation on the spatial distribution of birds in suburbs and reserves. - Diversity and Distributions 19(3): 294-306. DOI: 10.1111/j.1472-4642.2012. 00937.x

Ikin, K., Le Roux, D. S., Rayner, L., Villaseñor, N. R., Eyles, K., Gibbons, P., Manning, A. D. \& Lindenmayer, D. B. 2015. Key lessons for achieving biodiversity-sensitive cities and towns. - Ecological Management and Restoration 16(3): 206-214. DOI: 10.1111/emr.12180.

Johnson, B. J., Munafo, K., Shappell, L., Tsipoura, N., Robson, M., Ehrenfeld, J. \& Sukhdeo, M. V. 2012. The roles of mosquito and bird communities on the prevalence of West Nile virus in urban wetland and residential habitats. - Urban Ecosystems 15(3): 513-531. DOI: 10.1007/s11252-012-0248-1

Johnson, T. V. 2009. Review of diesel emissions and control. - International Journal of Engine Research 10(5): 275-285. DOI: 10.1243\%2F14680874JER04009

Jokimäki, J. 1999. Ocurrence of breeding bird species in urban parks: effects of park structure and broad-scale variables. - Urban Ecosystem 3: 21-34 DOI: 10.1023/A:1009505418327

Khan, S. I. \& Naher, H. 2009. Birds in Kurigram district of Bangladesh. - Journal of Threatened Taxa 1(4): 245-250. DOI: 10.11609/JoTT.o1698.245-50

Kocian, L., Némethová, D., Melicherová, D. \& Matušková, A. 2003. Breeding bird communities in three cemeteries in the City of Bratislava (Slovakia). - Folia Zoologica 52: 177-188. 
Lim, C. \& Sodhi, N. S. 2004. Responses of avian guilds to urbanisation in a tropical city. - Landscape and Urban Planning 66(4): 199-215. DOI: 10.1016/S0169-2046(03)00111-7

Lussenhop, J. 1977. Urban cemeteries as bird refuges. - The Condor 79(4): 456-461. DOI: 10.2307/1367725

MacGregor-Fors, I. \& Schondube, J. E. 2011. Gray vs. green urbanization: relative importance of urban features for urban bird communities. - Basic and Applied Ecology 12(4): 372-381. DOI: 10.1016/j.baae.2011.04.003

MacGregor-Fors, I., Morales-Pérez, L. \& Schondube, J. E. 2010. Migrating to the city: Responses of neotropical migrant bird communities to urbanization. - The Condor 112: 711-717. DOI: 10.1525/cond.2010.100062

MacGregor-Fors, I., Ortega-Álvarez, R. \& Schondube, J. E. 2009. On the ecological quality of urban systems: An ornithological perspective. - In: Graber, D. S. \& Birmingham, K. A. (eds.) Urban planning in the $21^{\text {st }}$ century. - New York, Nova Science Publishing, pp. 51-66.

Majer, J. D., Recher, H. F. \& Postle, A. C. 1994. Comparison of arthropod species richness in New South Wales and Western Australia canopies: a contribution to the species number debate. - Memoirs - Queensland Museum 36(1): 121-131.

Marzluff, J. M., Bowman, R. \& Donnelly, R. 2001. A historical perspective on urban bird research: trends, terms, and approaches. - In: Marzluff, J. M., Bowman, R. \& Donnelly, R. (eds.) Avian Ecology and Conservation in an Urbanizing World. - Springer, Boston, MA, pp. 1-17. DOI: 10.1007/978-1-4615-1531-9_1

Mazumdar, S., Ghose, D. \& Saha, G. K. 2016. Foraging strategies of Black Kites (Milvus migrans govinda) in urban garbage dumps. - Journal of Ethology 34(3): 243-247. DOI: 10.1007/s10164-016-0469-5.

Mazumdar, S., Ghose, D. \& Saha, G. K. 2018. Offal dumping sites influence the relative abundance and roosting site selection of Black Kites (Milvus migrans govinda) in urban landscape: a study from Kolkata metropolis, India. - Environmental Monitoring and Assessment 190: 20. DOI: 10.1007/s10661-017-6391-7

Mazumdar, S., Ghose, D. \& Saha, G. K. 2019. Assessment of offal consumption by Black Kites (Milvus migrans govinda) from urban garbage dumps through cafeteria experiment. - Acta Ornithologica 53(2): 163-172. DOI: 10.3161/00016454AO2018.53.2.006

McKinney, M. L. 2006. Urbanization as a major cause of biotic homogenization. - Biological Conservation 127(3): 247-260. DOI: 10.1016/j.biocon.2005.09.005

Miller, R. W. 1997. Urban Forestry: Planning and Managing Urban Green Spaces. $2^{\text {nd }}$ ed. - New Jersey: Prentice Hall, Inc., Upper Saddle River

Milton, K. 2002. Loving Nature: Towards an Ecology of Emotion. - Routledge, New York

Montgomery, D. C. \& Peck, E. 1992. Introduction to Linear Regression Analysis. $2^{\text {nd }}$ ed. - Wiley, New York

Mortberg, U. \& Wallentinus, H. G. 2000. Red-listed forest bird species in an urban environment - Assessment of green space corridors. - Landscape Urban Planning 50(4): 215-226. DOI: 10.1016/S0169-2046(00)00090-6

Nielsen, A. B., van den Bosch, M., Maruthaveeran, S. \& van den Bosch, C. K. 2014. Species richness in urban parks and its drivers: a review of empirical evidence. - Urban Ecosystems 17(1): 305-327. DOI: 10.1007/ s11252-013-0316-1

Olagunju, T. E. 2015. Impact of human induced deforestation, forest degradation and fragmentation on food security. - New York Science Journal 8(1): 4-16. Retrieved from: http://www.sciencepub.net/newyork/ ny080115/002_27606ny080115_4_16.pdf

Ortega-Álvarez, R. \& MacGregor-Fors, I. 2009. Living in the big city: Effects of urban land-use on bird community structure, diversity, and composition. - Landscape and Urban Planning 90(3-4): 189-195. DOI: 10.1016/j.landurbplan.2008.11.003

Paker, Y., Yom-Tov, Y., Alon-Mozes, T. \& Barnea, A. 2014. The effect of plant richness and urban garden structure on bird species richness, diversity and community structure. - Landscape and Urban Planning 122: 186-195. DOI: 10.1016/j.landurbplan.2013.10.005

Recher, H., Majer, J. D. \& Ganesh, S. 1996. Eucalypts, arthropods and birds: on the relation between foliar nutrients and species richness. - Forest Ecology and Management 85(1-3): 177-195. DOI: 10.1016/S03781127(96)03758-9

Sandström, U. G., Angelstam, P. \& Mikusinski, G. 2006. Ecological diversity of birds in relation to the structure of urban green space. - Landscape and Urban Planning 77: 39-53. DOI: 10.1016/j.landurbplan.2005.01.004

Seto, K. C., Güneralp, B. \& Hutyra, L. R. 2012. Global forecasts of urban expansion to 2030 and direct impacts on biodiversity and carbon pools. - Proceedings of the National Academy of Sciences 109(40): 1608316088. DOI: 10.1073/pnas. 1211658109

Shwartz, A., Turbé, A., Julliard, R., Simon, L. \& Prévot, A. C. 2014a Outstanding challenges for urban conservation research and action. - Global Environmental Change 28: 39-49. DOI: 10.1016/j. gloenvcha.2014.06.002. 
Shwartz, A., Turbé, A., Simon, L. \& Julliard, R. 2014b Enhancing urban biodiversity and its influence on citydwellers: An experiment. - Biological Conservation 171: 82-90. DOI: 10.1016/j.biocon.2014.01.009

Sol, D., Bartomeus, I., González-Lagos, C. \& Pavoine, S. 2017. Urbanisation and the loss of phylogenetic diversity in birds. - Ecology Letters 20(6): 721-729. DOI: 10.1111/ele.12769.

Sutherland, W. J. (ed.) 2006. Ecological census techniques: a handbook. - Cambridge University Press

Veech, J. A., Small, M. F. \& Baccus, J. T. 2010. The effect of habitat on the range expansion of a native and an introduced bird species. - Journal of Biogeography 38(1): 69-77. DOI: 10.1111/j.1365-2699.2010. 02397.x.

Villaseñor, N. R., Driscoll, D. A., Gibbons, P., Calhoun, A. J. K. \& Lindenmayer, D. B. 2017. The relative importance of aquatic and terrestrial variables for frogs in an urbanizing landscape: key insights for sustainable urban development. - Landscape and Urban Planning 157: 26-35. DOI: 10.1016/j. landurbplan.2016.06.006

White, J. G., Antos, M. J., Fitzsimons, J. A. \& Palmer, G. C. 2005. Non-uniform bird assemblages in urban environments: the influence of streetscape vegetation. - Landscape and urban planning 71(2-4): 123-135. DOI: 10.1016/j.landurbplan.2004.02.006

Yuan, Y. J., Zeng, G. M., Liang, J., Li, X. D., Li, Z. W., Zhang, C., Huang, L., Lai, X., Lu, L. H., Wu, H. P. \& Yu, X. 2014. Effects of landscape structure, habitat and human disturbance on birds: a case study in East Dongting Lake wetland. - Ecological Engineering 67: 67-75. DOI: 10.1016/j.ecoleng.2014.03.012

Zuur, A. F., Hilbe, J. M. \& Ieno, E. N. 2013. A Beginner's Guide to GLM and GLMM with R. - Highland Statistics Ltd., Newburgh

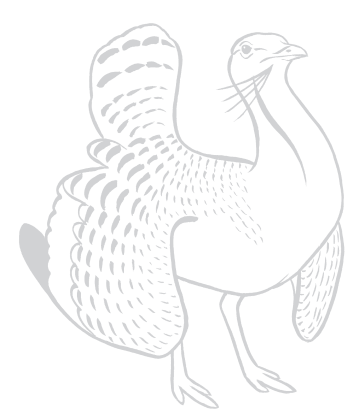

